\title{
The Design for Automatic Target Reporting System based on Image Processing
}

\author{
XU Zhihui, LI Weizhong, XIAO Yongjun* \\ HuBei Engineering University \\ School of Physics and Electronic Information Engineering \\ Xiaogan, China \\ E-mail: 75694676@qq.com
}

\begin{abstract}
Photo-electricity target with low cost, high efficiency and high reliability is widely used for gunnery training in military affairs and athletic sports. The automatic target reporting system based on image processing is constructed and some experimental is done. The result is shown that compared to the way of manual target, automatic target reporting system has the character of easy to operate, low-cost and easy to popularize.
\end{abstract}

Keywords-component; Photo-electricity target; impact point detecting; image process

\section{INTRODUCTION}

Compared to the conventional way of manual target, Photo-electricity target with high efficiency, high reliability is widely used for gunnery training in military affairs and athletic sports $^{[1][2][3][4]}$. Impact point detecting, as a function with a weapon's sighting system, is a key part for photoelectricity target, and its detecting precisions are an important index for the performance of photo-electricity target $^{[5][6]}$.

Recently, with the development of image process technology, automatic reporter based on optoelectronic coordinate positioning technology is an important research area for institutional repository at home and abroad ${ }^{[2]}[3][6]$. So the automatic target reporting system, based on the technology of image processing and digital signal processor, is designed in this paper. In the first part, the system hardware design is detailed; and in the second part, the software flow chart for designed system is described; in the third part, the experiment device is constructed, some experiment is done and some conclusion is derived from the experiment result.

\section{THE HARDWARE DESIGN FOR THE SYSTEM}

\section{A. The discussion for the designed whole system}

The system block diagram is shown in fig 1 , and it is consisted of control part, automatic shooting part and impact point detecting part. Where the control part, including microcontroller STM32F103ZE ${ }^{[7]}$, display module, keyboard and voice broadcast module, is used for timely receiving and displaying the result of impact point, and send command to driving the $\mathrm{x}$ and $\mathrm{y}$ motor to finish shooting action. The impact point detecting module is composed of CCD and the image processor which is a digital signal processor of TMS320DM642. The automatic shooting part is used for finish shooting task.

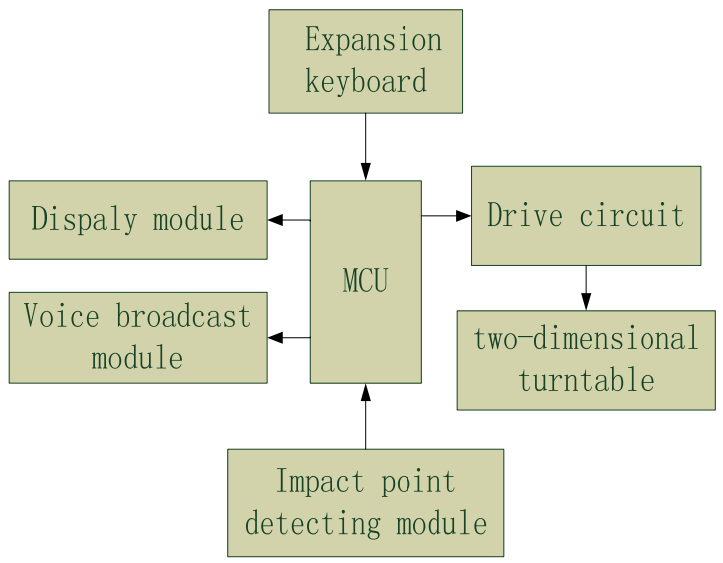

Figure 1 the system block diagram

For the impact point detecting module, analog PAL video stream is timely outputted from the image sensor CCD and converted into BT565 digital video format through the IC chip of SAA7115, then it is received and processed by a typical TI C6000 series DSP image processor of TMS320DM642. Through a series of operation such as image filtering, adaptive threshold segmentation, mass centre positioning method, the laser spot centroid coordinates is extracted out and sent to the control core STM32F103ZE through the RS232 serial port. Finally, it is changed into accordingly number of the photosensitive rings.

\section{B. The descripition of the control core STM32F103}

The STM32F103 family incorporates the highperformance ARM ${ }^{\circledR}$ Cortex ${ }^{\mathrm{TM}}$-M3 32-bit RISC core operating at a $72 \mathrm{MHz}$ frequency, high-speed embedded memories (Flash memory up to 512 Kbytes and SRAM up to 64 Kbytes), and an extensive range of enhanced I/Os and peripherals connected to two APB buses. All devices offer three 12-bit ADCs, four general purpose 16-bit timers plus two PWM timers, as well as standard and advanced communication interfaces: up to two I2Cs, three SPIs, two I2Ss, one SDIO, five USARTs, an USB and a CAN. It is suitable for a wide range of applications: motor drive and application control, PC peripherals gaming and GPS platforms. 


\section{THE SOFTWARE DESIGN FOR THE SYSTEM}

The system flow chart is shown in fig 2. When the electric is switch on, the system enter into initialed states including serial port initialed, display module initialed, voice broadcast module initialed as well as image processor initialed, and then enter into next state to wait external commands. If the number of photosensitive rings is setup and shooting command is sent via the expand keyboard. The microcontroller STM32F103ZE controls the auto shooting device to emit the laser, and at the same time, the impact point is detected out by image processor, and sent to the microcontroller. If it hit the target, the shooting action is finish and the system return to wait state; otherwise, the shooting device is adjusted to reshooting.

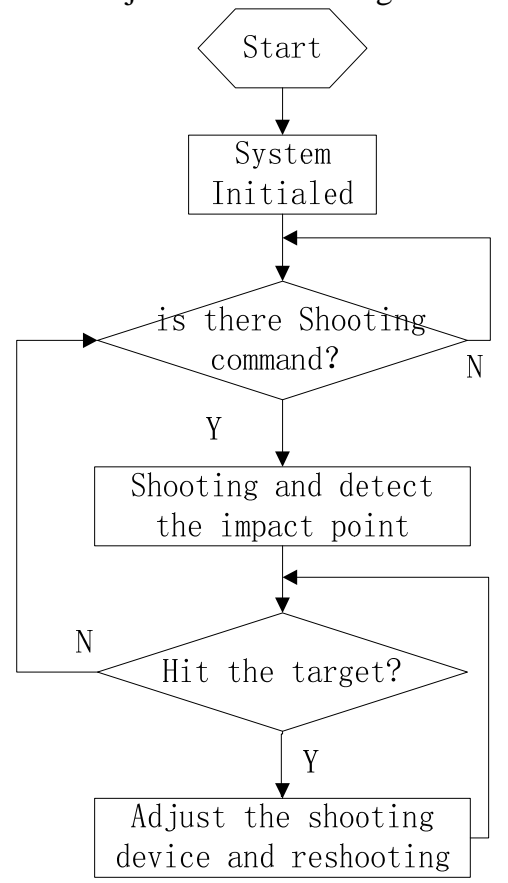

Figure 2 the flow chart for the system software

\section{EXPERIMENT AND RESULT ANALYSIS}

In order to check the performance of this system, the experiment device is constructed and its schematic diagram is illustrated in figure 3 and the physical diagrams for the Experiment system is figure 4, and the picture of whole experiment set is shown in figure 5 . On the basis of this experiment system, some experiment is done, and the test condition is shown in table1. The test instrument is DS1052E series digital storage oscilloscope, Rohde\&Schwarz SMT03 signal generator, DT99208 Digital multi-meter and meter stick. The test environment is in the room with strong light on the daylight, and the test distance is 3 meters between photo-electricity targets to laser chaingun, and each test is redo for ten times.

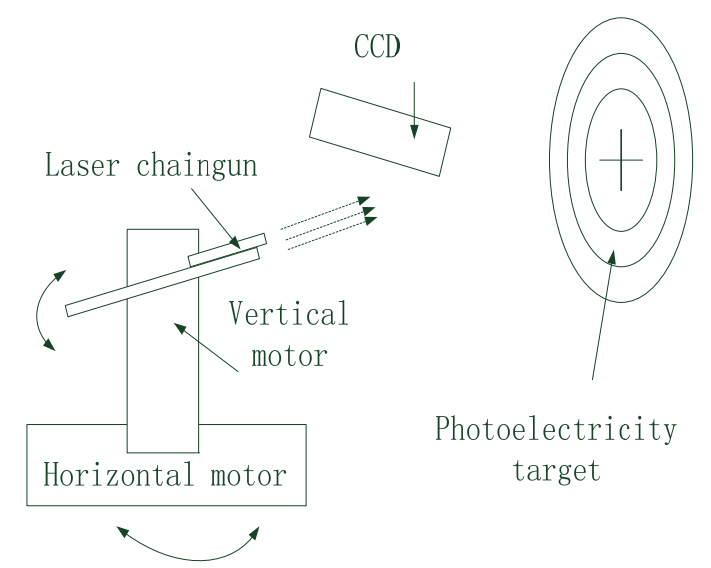

Figure 3 the schematic diagram for experiment device

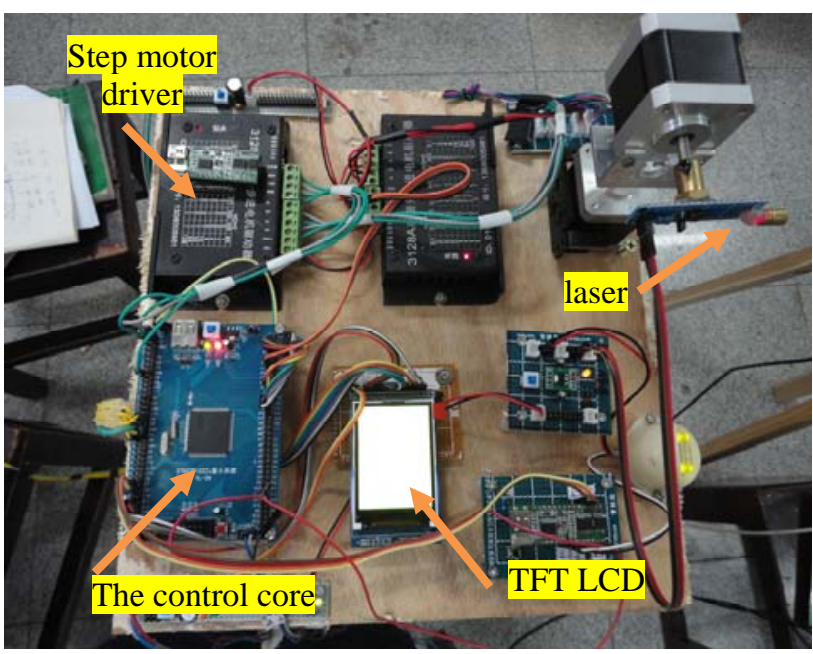

Figure 4 Physical diagrams for the Experiment system

The experimental result is illustrated in table 2, the test data is the distance between impact point and ring center for each rings. As the distance for each ring is 5 centimeter, so it hit the ten-point ring when the impact point is less than 5 centimeter. Similarly, when the impact point is less than 10 centimeter but more than 5 centimeter, it hit the nine-point ring. From the table 2, we can infer that each shooting can hit the target and according rings.

Table 1 experiment condition

\begin{tabular}{|c|l|}
\hline item & \multicolumn{1}{|c|}{ Speciation } \\
\hline $\begin{array}{c}\text { Test } \\
\text { instrument }\end{array}$ & $\begin{array}{l}\text { DS1052E series digital storage } \\
\text { oscilloscope, Rohde\&Schwarz SMT03 } \\
\text { signal generator,DT99208 Digital } \\
\text { multi-meter and meter stick }\end{array}$ \\
\hline Test area & Indoor with strong light \\
\hline Test distance & $\begin{array}{l}\text { 3 meters(from photo-electricity target } \\
\text { to laser chaingun) }\end{array}$ \\
\hline $\begin{array}{c}\text { photo- } \\
\text { electricity } \\
\text { target }\end{array}$ & $\begin{array}{l}\text { The distance for each ring is 5 } \\
\text { centimeters }\end{array}$ \\
\hline
\end{tabular}


Table 2 experiment result

\begin{tabular}{|c|c|c|c|c|c|c|}
\hline \multirow{2}{*}{ number } & \multicolumn{6}{|c|}{ For each ring, The distance between impact } \\
& \multicolumn{7}{|c|}{ point and ring center (cm) } \\
\cline { 2 - 7 } & 5 & 6 & 7 & 8 & 9 & 10 \\
\hline 1 & 28 & 22 & 17 & 12.3 & 7.8 & 0.2 \\
\hline 2 & 28.1 & 22.7 & 17.7 & 13 & 9.2 & 0.6 \\
\hline 3 & 27.5 & 21.6 & 16.5 & 13 & 9 & 0.7 \\
\hline 4 & 27.6 & 21.1 & 17.0 & 12 & 7.7 & 1.7 \\
\hline 5 & 26.9 & 21.9 & 18.1 & 11.5 & 6.7 & 4 \\
\hline 6 & 27 & 22.5 & 17.6 & 13.5 & 6.5 & 1.1 \\
\hline 7 & 27.3 & 22.6 & 18 & 14 & 7.5 & 1 \\
\hline 8 & 27.7 & 23 & 16.8 & 11.7 & 5.8 & 0.2 \\
\hline 9 & 26.8 & 23.6 & 17.5 & 12.5 & 8 & 0.5 \\
\hline 10 & 27 & 22.9 & 17.3 & 12.6 & 8.4 & 1.4 \\
\hline mean & 27.39 & 22.39 & 17.35 & 12.73 & 7.66 & 0.78 \\
\hline
\end{tabular}

\section{CONCLUSIONS}

The automatic target reporting technology is a practical technology for shooting training in military affairs and athletic sports. Compared with the way of manual target, it has the advantages in accuracy and reliability. So the automatic target reporting system, based on the technology of image processing and digital signal processor, is designed in this paper. On the basis of this, some experiment is done and the result is also analyzed. The conclusions derived from experiment data are as follows:

- For the Photo-electricity target based on image processing, each shooting can hit the target and hit the according rings.
- Compared to the way of manual target, automatic target reporting system has the character of easy to operate, low-cost and easy to popularize.

In this experiment, some problems is exposed out, such as bias light can seriously affect the detecting precision for photo-electricity target, this point out ours further next research direction.

\section{ACKNOWLEDGMENT}

We would like to express our thanks to the professor $\mathrm{s}$ Xinming Wang and ours students for their valuable discussions and helps to improve this paper and ready for our experiment.

\section{REFERENCES}

[1] David R. Parker, Steven C. Gustafson. Unified measures of target detection system performance evaluation. SPIE, 2006, 6237:62370M1-62370M11

[2] Craig R. Schwartz, Jack N. Cederquist, Michael T. Eismann. Target detection using infrared spectral sensors. SPIE, 1996, 2819:182-194

[3] Abhijit Mahalanobit, Robert R. Muise, S.Robert Stanfill, et.al. Elements of target detection for ground sensor systems. SPIE, 2005, 5796:155-166

[4] C.C. Chang. Detecting target velocity and location using a novel optoelectronic sensing system. SPIE, 2004, 5612:362-369

[5] The application report of The TMS320DM642 video Port MiniDriver [Z].Texas Instruments. August 2003

[6] TMS320C6000 DSP GPIO reference guide [Z]. Texas Instruments. March 2004

[7] TMS320DM642 video/imaging fixed-point digital signal processor data manual $[\mathrm{EB} / \mathrm{OL}]$.

[8] http://focus.ti.com/lit/ds/symlink/tms320dm642.pdf , 2010-3-21

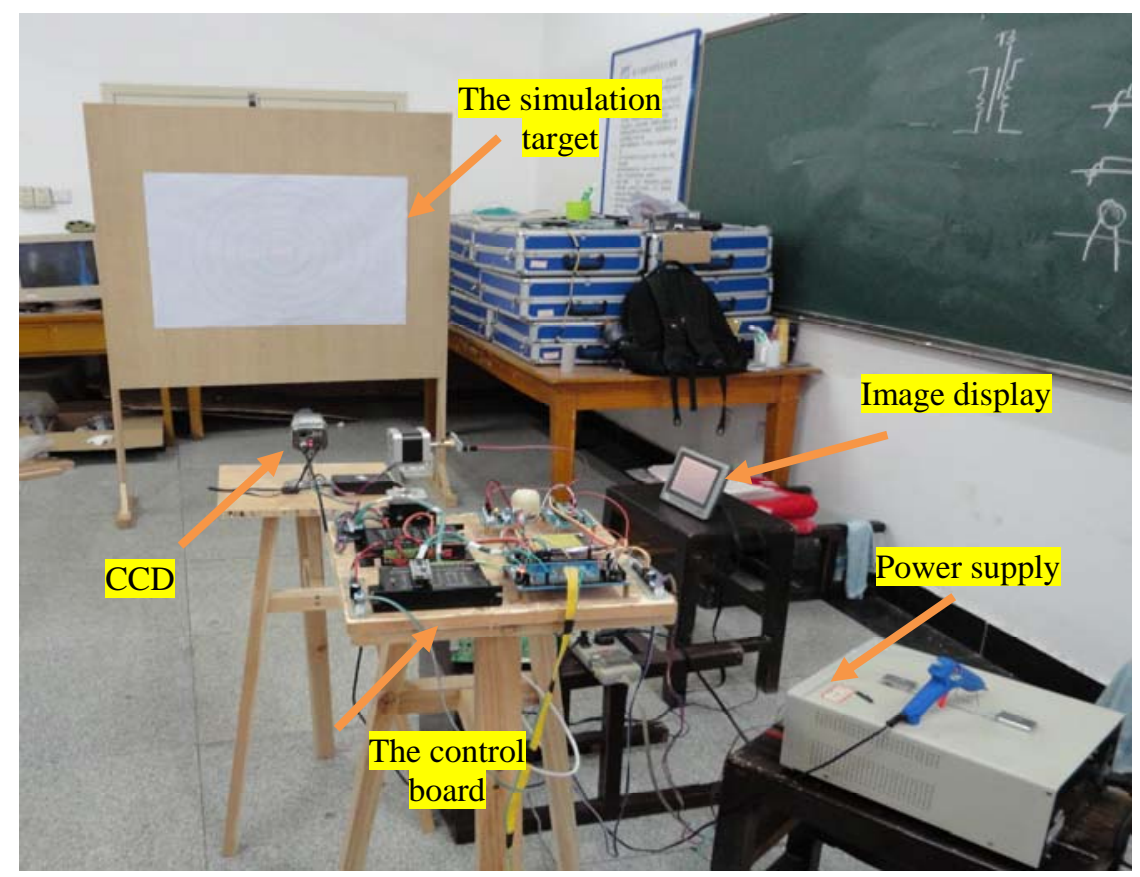

Figure 5 the picture of whole experiment set 